\title{
THE INDEPENDENCE OF THE NOTIONS OF HOPFIAN AND CO-HOPFIAN ABELIAN P-GROUPS
}

\author{
GÁBOR BRAUN AND LUTZ STRÜNGMANN
}

(Communicated by Birge Huisgen-Zimmermann)

\begin{abstract}
Hopfian and co-Hopfian Abelian groups have recently become of great interest in the study of algebraic and adjoint entropy. Here we prove that the existence of the following three types of infinite abelian $p$-groups of size less than $2^{\aleph_{0}}$ is independent of ZFC: (a) both Hopfian and co-Hopfian, (b) Hopfian but not co-Hopfian, (c) co-Hopfian but not Hopfian. All three types of groups of size $2^{\aleph_{0}}$ exist in ZFC.
\end{abstract}

\section{INTRODUCTION}

A very simple result from linear algebra states that an endomorphism of a finite dimensional vector space is injective if and only if it is surjective and hence an isomorphism. However, an infinite dimensional vector space never has this property, as the standard shift endomorphisms show.

Reinhold Baer [B] was the first to investigate these properties for Abelian groups rather than vector spaces. While he used the terminology $Q$-group and $S$-group, researchers now use the notions of Hopfian group and co-Hopfian group. These groups have arisen recently in the study of algebraic entropy and its dual, adjoint entropy; see e.g., DGSZ,GG, which are the motivations for our work.

Recall that an (Abelian) group $G$ is Hopfian if every epimorphism $G \rightarrow G$ is an automorphism. Dually, $G$ is said to be co-Hopfian if every monomorphism $G \rightarrow G$ is an automorphism.

Some obvious examples: finite groups are both Hopfian and co-Hopfian, the group of integers is Hopfian but not co-Hopfian, and the Prüfer group $\mathbb{Z}\left(p^{\infty}\right)$ is co-Hopfian but not Hopfian. Hence the notions are independent of each other. However, the existence of infinite co-Hopfian reduced $p$-groups was first established by Crawley $\mathrm{Cr}$.

As it is often the case in Abelian group theory, one attacks a problem by looking at three different cases, namely, the torsion case, the torsion-free case and the mixed case. Here we will concentrate on the $p$-groups, but for the convenience of

Received by the editors February 1, 2012 and, in revised form, May 3, 2013, September 17, 2013 and November 11, 2013.

2010 Mathematics Subject Classification. Primary 20K30; Secondary 20K15.

Key words and phrases. Martin's axiom, Hopfian groups, co-Hopfian groups.

The first author's research was partially supported by the Hungarian Scientific Research Fund, Grant No. NK 81203 and project STR 627/1-6 of the German Research Foundation DFG. 
the reader we list some easy observations about (co-)Hopfian groups in general (see also [GG2]).

- A torsion-free group is co-Hopfian if and only if it is divisible of finite rank; i.e., it is isomorphic to a finite dimensional $\mathbb{Q}$-space.

- A torsion-free group of finite rank is Hopfian.

- Finitely generated groups are Hopfian, and dually, finitely co-generated groups (like $\mathbb{Z}\left(p^{\infty}\right)$ ) are co-Hopfian.

- Every group $G$ with endomorphism $\operatorname{ring} \operatorname{End}(G) \cong \mathbb{Z}$ is Hopfian; thus arbitrarily large Hopfian groups exist.

- Reduced Hopfian or co-Hopfian p-groups are semi-standard and so have cardinality at most $2^{\aleph_{0}}$;

- Reduced countable Hopfian or co-Hopfian p-groups are finite.

Inspired by the last two statements, Goldsmith and Gong asked in GG2 if the notions of Hopficity and co-Hopficity are independent at all for reduced $p$-groups and if there is a Hopfian and/or co-Hopfian group of size $\aleph_{1}$ if the continuum hypothesis fails. Note that there are Hopfian and co-Hopfian reduced $p$-groups of size the continuum. Here is our main theorem showing that the existence of such groups is independent of ZFC:

Theorem 1.1. The following statements are consistent with $Z F C$ even if the continuum $2^{\aleph_{0}}$ is arbitrarily large:

(1) For every prime $p$ there are reduced p-groups of size $\aleph_{1}$ of the following three types:

(a) both Hopfian and co-Hopfian,

(b) Hopfian but not co-Hopfian,

(c) co-Hopfian but not Hopfian.

(2) For every prime p every reduced infinite p-group of size strictly less than the continuum is neither Hopfian nor co-Hopfian.

Remark 1.2. There always exist continuum-sized reduced $p$-groups of types (1.a) (1.b) and (1.c) in ZFC.

The proof of this statement uses rather technical but old and classic ideas; cf. $\mathrm{Cr}$. As usual one kills unwanted endomorphisms by adding new elements during the construction, establishing Lemmas 2.1 and 3.1 for the groups. One then finishes as below.

Actually, as we shall see, (1) holds in the forcing extension of the universe $\mathfrak{M}$ by $\operatorname{Fin}\left(\omega_{1}, 2\right)$, i.e., the partial poset of partial maps $q: \omega_{1} \rightarrow\{0,1\}$ with finite domain $\operatorname{dom}(q) \subseteq \omega_{1}$. Furthermore, (2) follows from Martin's axiom, even from its weak version $\mathrm{MA}(\sigma$-centered $)$.

We prove the first part of the theorem in Sections [2] and 3. In the former section we will construct two Hopfian groups: a co-Hopfian one and a non-co-Hopfian one. In the latter section we carry out a similar construction of a co-Hopfian group.

Finally, in Section 4 we prove the second part of the theorem via constructing endomorphisms.

In the sequel the word group shall always mean an additively written Abelian group; the book $[\mathrm{F}$ ] shall serve as a reference to ideas needed in Abelian group theory. 


\section{A Hopfian but nOt CO-Hopfian group}

In this section we construct Hopfian groups in the forcing extension of the universe $\mathfrak{M}$ by $\operatorname{Fin}\left(\omega_{1}, 2\right)$. Actually, it will be more convenient to consider the equivalent forcing poset $P=\operatorname{Fin}\left(\omega_{1} \times \mathbb{Z}^{+}, 2\right)$, i.e., the partial poset of partial maps $q: \omega_{1} \times \mathbb{Z}^{+} \rightarrow\{0,1\}$ with finite domain. Thus we work in $\mathfrak{M}[F]$, where $F$ is a $P$-generic filter over $\mathfrak{M}$. Here we have new functions $\eta_{\alpha}: \mathbb{Z}^{+} \rightarrow\{0,1\}$ added to the universe for $\alpha<\omega_{1}$.

Let us construct some subgroups of $C=t\left(\prod_{n=1}^{\infty} \mathbb{Z}_{p^{n}} e_{n}\right)$ for some fixed prime $p$. Let $x$ be the monomorphism defined informally by $e_{k} x:=p e_{k+1}$ for every $k \in \mathbb{N}$, i.e., $\left(\sum_{k=1}^{\infty} n_{k} e_{k}\right) x:=\sum_{k=1}^{\infty} p n_{k} e_{k+1}$ in general. Moreover, we put

$$
a_{\alpha, n}:=\sum_{k}^{\infty} \eta_{\alpha}(k) p^{k-n} e_{k} \quad \text { for } \alpha<\omega_{1}, n \in \mathbb{Z}^{+},
$$

hence

$$
a_{\alpha, n}=\eta_{\alpha}(n) e_{n}+p a_{\alpha, n+1} \quad \text { for } \alpha<\omega_{1}, n \in \mathbb{Z}^{+} \text {. }
$$

We define

$$
\begin{aligned}
G_{h, c} & :=\left\langle e_{n}, a_{\alpha, n}: n=1, \ldots, \infty, \alpha<\omega_{1}\right\rangle, \\
G_{h} & :=\left\langle e_{n}, a_{\alpha, n} x^{k}: k=0, \ldots, \infty, n=1, \ldots, \infty, \alpha<\omega_{1}\right\rangle .
\end{aligned}
$$

A common basic subgroup of $C, G_{h, c}$ and $G_{h}$ is

$$
B=\bigoplus_{n=1}^{\infty} \mathbb{Z}_{p^{n}} e_{n}
$$

because it is a pure cyclic subgroup and factoring with it leads to divisible quotients. The divisibility of $G_{h, c} / B$ and $G_{h} / B$ follows from (2.2).

We first determine the endomorphism rings of $G_{h, c}$ and $G_{h}$. Recall that a homomorphism $\phi: A \rightarrow X$ between two Abelian $p$-groups is called small if for all $k>0$ there is an $n \geq 0$ such that $p^{n} A\left[p^{k}\right] \phi=0$. By $\widehat{X}$ we denote the completion of $X$ in $p$-adic topology.

\section{Lemma 2.1.}

$$
\begin{aligned}
\text { End } G_{h, c} & =J_{p} \oplus \operatorname{small}\left(G_{h, c}\right), \\
\text { End } G_{h} & =\widehat{J_{p}[x]} \oplus \operatorname{small}\left(G_{h}\right) .
\end{aligned}
$$

Proof. First note that the groups $G_{h, c}$ and $G_{h}$ are pure $p$-torsion subgroups of $C$; hence every homomorphism between these groups is uniquely determined on the basis elements $e_{n}$. Therefore a common generalization of the results stated in the lemma is

$$
\operatorname{Hom}\left(G_{h, c}, G_{h}\right)=\widehat{J_{p}[x]} \oplus \operatorname{small}\left(G_{h, c}, G_{h}\right) .
$$

In other words, for every homomorphism $f$ and every positive integer $N$, there is a polynomial $k^{\prime} \in \mathbb{Z}[x]$ making $f-k^{\prime}$ small on $G_{h, c}\left[p^{N}\right]$. We are now going to prove this.

For simplicity, we abuse notation, and let $f$ also denote a name of the homomorphism $G_{h, c} \rightarrow G_{h}$. Let $N \in \mathbb{N}$. 
There are conditions $r_{i, j}$ in the generic filter $F$ forcing the $e_{j}$-coordinate of $e_{i} f$ to be some $k_{i, j} \in \mathbb{Z}_{p^{j}}$. The $r_{i, j}$ altogether have countable domain, which is therefore disjoint from $\{\alpha\} \times \mathbb{Z}^{+}$for some $\alpha \in \omega_{1}$.

There is an $r$ in the generic filter $F$ forcing that $f$ is a homomorphism $G_{h, c} \rightarrow G_{h}$, and

$$
a_{\alpha, N} f=g+k a_{\alpha, M}+\sum_{i=1}^{l} k_{i} a_{\alpha_{i}, M_{i}}
$$

for some $g \in B$, polynomials $k, k_{i} \in \mathbb{Z}[x]$ and pairwise distinct ordinals $\alpha, \alpha_{i}<\omega_{1}$.

For all positive integers $n \geq N$ where $(\alpha, n)$ is not in the domain of $r$, let us consider the automorphism $\varphi$ of the forcing notion switching the value of $\eta_{\alpha}$ at $n$; i.e., $\eta_{\alpha}^{\varphi}$ coincides with $\eta_{\alpha}$ except at the place $n$ where $\eta_{\alpha}^{\varphi}(n)=1-\eta_{\alpha}(n)$. For $\beta \neq \alpha$, the function $\eta_{\beta}$ remains unchanged: $\eta_{\beta}^{\varphi}=\eta_{\beta}$.

On the level of partial functions, for every partial function $q$, we have $\operatorname{dom} q^{\varphi}=$ $\operatorname{dom} q$ and $(\beta, m) q^{\varphi}=(\beta, m) q$ for $(\beta, m) \in \operatorname{dom} q$ and $(\beta, m) \neq(\alpha, n)$. The crucial change is $(\alpha, n) q^{\varphi}=1-(\alpha, n) q$ whenever $(\alpha, n) \in \operatorname{dom} q$. In particular, $\varphi$ leaves $r$ and the $r_{i, j}$ invariant.

Now applying the automorphism $\varphi$, we obtain, since $r$ forces it, that $f^{\varphi}$ is again a homomorphism $G_{h, c} \rightarrow G_{h}$ satisfying

$$
\left(a_{\alpha, N} \pm p^{n-N} e_{n}\right) f^{\varphi}=g+k\left(a_{\alpha, M} \pm p^{n-M} e_{n}\right)+\sum_{i=1}^{l} k_{i} a_{\alpha_{i}, M_{i}} .
$$

Also, the $e_{j}$-coordinate of $e_{i} f^{\varphi}$ is $k_{i, j}$, because $r_{i, j}$ forces it, so $f^{\varphi}=f$ since the two maps agree on the $e_{n}$. Hence the difference of the two equations (2.9) and (2.10) simplifies to

$$
p^{n-N} e_{n} f=p^{n-M} k e_{n}
$$

(we repeat) for all but finitely many $n \geq N$ (those for which $(\alpha, n)$ is not in support of $p$ ).

It follows that $0=p^{n} e_{n} f=p^{n+N-M} k e_{n}$ for almost all $n$. Therefore $p^{n+N-M} k$ must be divisible by $p^{n}$, which means that the polynomial $k^{\prime}:=p^{N-M} k$ has integral coefficients; thus (2.11) beautifies to

$$
p^{n-N} e_{n} f=p^{n-N} k^{\prime} e_{n}
$$

for all but finitely many $n$.

Finally, we can conclude that $f-k^{\prime}$ is zero on all but finitely many of the $p^{n-N} e_{n}$, hence $f-k^{\prime}$ is small on $G_{h, c}\left[p^{N}\right]$.

It remains to show that the discovered endomorphism rings ensure that the groups $G_{h, c}$ and $G_{h}$ are Hopfian. We thank the referee for suggesting the Theorem of Irwin and Walker to shorten our original proof.

Proposition 2.2. Let $G$ be a semi-standard reduced abelian p-group with a strictly height-increasing monomorphism $x$, i.e., $h(g x)>h(g)$ for all $g \in G \backslash\{0\}$. Assume End $G=\widehat{J_{p}[x]} \oplus \operatorname{small}(G)$. Then $G$ is Hopfian but not co-Hopfian.

Proof. Obviously, $G$ is not co-Hopfian, as $x$ is a monomorphism which is not an isomorphism. To prove $G$ is Hopfian, let $f: G \rightarrow G$ be an epimorphism. We will show that it is an isomorphism adapting $[\mathrm{P}, 16.14]$. 
If $f$ is small, then $G=f(G)=f(B)$, where $B$ is basic in $G$. Since $G$ is semi-standard, $B$ is countable, and so too is $G$. As a consequence of Zippins Theorem (see [F, Proposition 77.5]) one can write $G=\bigoplus_{i<\omega} G_{i}$ where $G_{i}$ is nontrivial. It follows that the canonical image of $\operatorname{End}(G)$ in $\operatorname{Hom}(G[p], G)$ has size the continuum: if $\delta_{n}: G_{n}[p] \rightarrow G$ is either the zero or the identity map on $G_{n}[p]$, then the vector $\underline{\delta}=\left(\delta_{0}, \delta_{1}, \cdots\right)$ represents a homomorphism: $G[p] \rightarrow G$. Observe that there are continuum many such homomorphisms. Now if $\epsilon_{n}: G_{n} \rightarrow G$ is the corresponding zero or identity map on $G_{n}$, then the vector $\underline{\epsilon}=\left(\epsilon_{0}, \epsilon_{1}, \cdots\right)$ represents a homomorphism: $G \rightarrow G$ such that $\underline{\epsilon}$ restricts to $\underline{\delta}$ on $G[p]$.

However, the images of both $\widehat{J_{p}[x]}$ and small $(G)$ are only countable: First, $\widehat{J_{p}[x]}=\mathbb{Z}[x]+p \widehat{J_{p}[x]}$ with the first summand countable, and the image of the second summand is clearly 0 . Second, every small endomorphism $\varphi$ restricted to $G[p]$ should be 0 on the cofinite subgroup $p^{n} G[p]$ for some $n$. This leaves only countably many possibilities for the restriction of $\varphi$ to $G[p]$. All in all, the images of $\widehat{J_{p}[x]}$ and $\operatorname{small}(G)$ in $\operatorname{Hom}(G[p], G)$ are countable, but the image of End $G$ is continuum, a contradiction.

Therefore $f$ is not small, hence it is of the form $f=\alpha+\phi$ where $0 \neq \alpha \in \widehat{J_{p}[x]}$ and $\phi \in \operatorname{small}(G)$.

Second, we want to show that $\alpha$ has an invertible constant term in several steps.

As the first step, we decompose $\alpha$ : let $\alpha=p^{k} \alpha^{\prime}$, where $p \nmid \alpha$. Treating $\alpha^{\prime}$ as a power series in $x$, let the $x^{j}$-term be the first one whose coefficient is not divisible by $p$; i.e., $\alpha^{\prime}=\beta x^{j}+p \gamma$, where $\beta x^{j}$ is the sum of terms of degree at least $j$ with $\beta$ having an invertible constant term, and $p \gamma$ is the sum of terms of $x$-degree less than $j$. In particular, $\beta$ is height-preserving, i.e., $h(g \beta)=h(g)$ for all $g \in G$, hence it is a monomorphism. It follows that

$$
\alpha=p^{k} x^{j} \beta+p^{k+1} \gamma, \quad \beta, \gamma \in \widehat{J_{p}[x]}, \operatorname{deg} \gamma<j .
$$

The second step is determining the kernel of $\alpha$ :

$$
\operatorname{ker} \alpha\left[p^{k+1}\right]=\operatorname{ker} p^{k} x^{j} \beta\left[p^{k+1}\right]=\operatorname{ker} p^{k} 1_{G}\left[p^{k+1}\right]=G\left[p^{k}\right]
$$

using that $x$ and $\beta$ are monomorphisms, and $p^{k+1} \gamma$ is 0 on $G\left[p^{k+1}\right]$. We conclude that

$$
\operatorname{ker} \alpha=G\left[p^{k}\right] \text {. }
$$

Since $\phi$ is small, there is a natural number $N$ such that

$$
p^{N} G\left[p^{k+1}\right] \subseteq \operatorname{ker} \phi .
$$

Let $n>N$ be arbitrary. As

$$
p^{n}\left(G\left[p^{n}\right] f^{-1}\right)\left[p^{k+1}\right] \subseteq \operatorname{ker} f \cap \operatorname{ker} \phi \subseteq \operatorname{ker} \alpha=G\left[p^{k}\right],
$$

we have $p^{n-1}\left(G\left[p^{n}\right] f^{-1}\right)\left[p^{k+2}\right] \subseteq G\left[p^{k+1}\right]$ (cf. [P, 16.1(d)]), hence

$$
p^{n-1}\left(G\left[p^{n}\right] f^{-1}\right)=p^{n-1}\left(G\left[p^{n}\right] f^{-1}\right)\left[p^{k+1}\right] .
$$

Consequently (cf. [P, 16.1(b)])

$$
\begin{gathered}
p^{n-1} G[p]=p^{n-1}\left(G\left[p^{n}\right]\right)=p^{n-1}\left(G\left[p^{n}\right] f^{-1}\right) f=p^{n-1}\left(G\left[p^{n}\right] f^{-1}\right)\left[p^{k+1}\right] f \\
=p^{n-1}\left(G\left[p^{n}\right] f^{-1}\right)\left[p^{k+1}\right] \alpha=p^{n-1}\left(G\left[p^{n}\right] f^{-1}\right)\left[p^{k+1}\right] p^{k} x^{j} \beta \subseteq p^{n-1+k+j} G[p],
\end{gathered}
$$


using that $f$ is epic, $\phi$ and $p^{k+1} \gamma$ are 0 on $p^{n-1}\left(G\left[p^{n]} f^{-1}\right)\left[p^{k+1}\right]\right.$, and that $x$ is strictly height-increasing.

So we have $p^{n-1} G[p] \subseteq p^{n-1+k+j} G[p]$ for all $n>N$. As $G$ is unbounded, this is only possible if $k=j=0$, i.e., $\alpha=\beta$. Therefore $\alpha$ is also height-preserving.

Finally, let $B=\bigoplus_{n=1}^{\infty} B_{n}$ be a basic subgroup of $G$ with $B_{n}$ a direct sum of cyclic groups of order $p^{n}$. Taking the $N$ from above (chosen to satisfy (2.16)), the canonical direct complement of $B_{1} \oplus \cdots \oplus B_{N}$ is $H_{N}:=\left\langle p^{N} G, B_{N+1}, B_{N+2}, \ldots\right\rangle$. Now on $H_{N}[p]=p^{N} G[p]$, the small map $\phi$ is 0 by (2.16), so $f$ coincides with $\alpha$ here. Hence $f$ is height-preserving on $H_{N}[p]=p^{N} G[p]$. It follows that $f$ is injective on $H_{N}$ and $H_{N} f$ is a direct complement of $B_{1} \oplus \cdots \oplus B_{N}$ by [IW] Theorem 16]. This leads to the commutative diagram

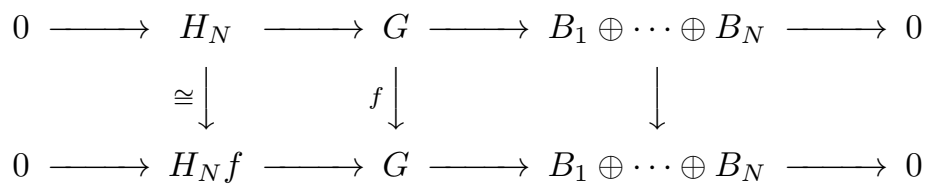

Since $f$ is epic, the right-most vertical arrow is epic. As the $B_{n}$ are finite, this arrow must be an isomorphism. By the Five Lemma, therefore, $f$ is an isomorphism.

Finally, we conclude from Lemma 2.1 and Proposition 2.2 that $G_{h}$ is Hopfian, while it is obviously not co-Hopfian. It also follows from Lemma 2.1 that $G_{h, c}$ is both Hopfian and co-Hopfian. Obviously, both groups have size $\aleph_{1}$, establishing the Hopfian parts (1.a) and (1.b) of Theorem 1.1] (1)

\section{A co-Hopfian but not Hopfian group}

We follow an analogous strategy as in the previous section to construct a coHopfian group which is not Hopfian. Therefore we outline only the differences.

This time we use the equivalent forcing poset $\operatorname{Fin}\left(\omega_{1} \times \mathbb{Z}^{+} \times \mathbb{Z}^{+}, 2\right)$, so the newly added functions are $\eta_{\alpha}: \mathbb{Z}^{+} \times \mathbb{Z}^{+} \rightarrow\{0,1\}$. Let $C=t\left(\prod_{n=1}^{\infty} \bigoplus_{m=1}^{n} \mathbb{Z}_{p^{n}} e_{n, m}\right)$ and put

$$
a_{\alpha, n}^{(i)}:=\sum_{k=n+i}^{\infty} \sum_{m=1}^{k} \eta_{\alpha}(k-i, m) p^{k-n} e_{k, m} \quad \text { for } \alpha<\omega_{1}, i \geq 0, n \geq 1 .
$$

We define

$$
G_{c}:=\left\langle e_{n, m}, a_{\alpha, n}^{(i)} x^{k}: n \geq m \geq 1, k, i \geq 0\right\rangle \subseteq C,
$$

where $x: C \rightarrow C$ is defined by

$$
\begin{aligned}
e_{n, n} x & :=0 \\
e_{n+1, m} x & :=e_{n, m} \quad m \leq n .
\end{aligned}
$$

Hence

$$
\begin{aligned}
a_{\alpha, n+1}^{(i+1)} x & =a_{\alpha, n}^{(i)}+\sum_{m=1}^{n+i} \eta_{\alpha}(n, m) p^{i} e_{n+i, m} \\
a_{\alpha, n}^{(i)} & =\sum_{m=1}^{n+i} \eta_{\alpha}(n, m) p^{i} e_{n+i, m}+p a_{\alpha, n+1}^{(i)} .
\end{aligned}
$$


Note that a common basic subgroup of $C$ and $G_{C}$ is

$$
B:=\bigoplus_{n=1}^{\infty} \bigoplus_{m=1}^{n} \mathbb{Z}_{p^{n}} e_{n, m}
$$

It is clear from the above that $x$ is an epimorphism of $C$ and $G_{c}$.

As in the last section we determine the endomorphism ring of $G_{c}$. Note that $C[p] x=0$, and hence the $(p, x)$-adic completion $J_{p}[[x]]$ of $\mathbb{Z}[x]$ acts on $C$.

\section{Lemma 3.1.}

$$
\text { End } G_{c}=J_{p}[[x]] \oplus \operatorname{small}\left(G_{c}\right) .
$$

Proof. The proof is analogous to that of Lemma 2.1, so we point out only the differences.

Now the reformulation of the statement reads: for every homomorphism $f$ and every positive integer $N$, there is a polynomial $k^{\prime} \in \mathbb{Z}[x]$ making $f-k^{\prime}$ small on $G_{c}\left[p^{N}\right]$.

This time the forcing argument provides an $\alpha$ such that

$$
a_{\alpha, N}^{(0)} f=g+k a_{\alpha, M}^{(i)}+\sum_{j=1}^{l} k_{j} a_{\alpha_{j}, M_{j}}^{\left(i_{j}\right)}
$$

for some $g \in B$, polynomials $k, k_{i} \in \mathbb{Z}[x]$, non-negative integer $i$ and pairwise distinct ordinals $\alpha, \alpha_{j}$. We may assume that the constant term of $k$ is not divisible by $p^{M}$ when $i>0$. Moreover (still by the forcing argument via switching $\eta_{\alpha}(n, m)$ ), for all but finitely many pairs $n, m$ of positive integers with $n \geq N$,

$$
\left(a_{\alpha, N}^{(0)} \pm p^{n-N} e_{n, m}\right) f=g+k\left(a_{\alpha, M}^{(i)} \pm p^{n+i-M} e_{n+i, m}\right)+\sum_{j=1}^{l} k_{j} a_{\alpha_{j}, M_{j}}^{\left(i_{j}\right)}
$$

with the convention that $e_{p, q}=0$ for $p<q$.

The difference of the two equations (3.9) and (3.10) reduces to

$$
p^{n-N} e_{n, m} f=k p^{n+i-M} e_{n+i, m}
$$

(we repeat) for all but finitely many pairs $n, m$ with $n \geq N$. If $i>0$, then choosing $m=n+i$ and $n$ large enough, we obtain $0=p^{n-N} e_{n, n+i} f=k p^{n+i-M} e_{n+i, n+i}$, contradicting that the constant term of $k$ is not divisible by $p^{M}$.

Hence $i=0$, and we finish as in Lemma 2.1 deriving that $f-k^{\prime}$ is small on $G_{c}\left[p^{N}\right]$, where $k^{\prime}:=p^{N-M} k$ is an integral polynomial.

Finally, we have to show that the above group is co-Hopfian.

Proposition 3.2. Let $G$ be a semi-standard reduced abelian p-group with an endomorphism $x$, which is 0 on $G[p]$. Assume End $G=J_{p}[[x]] \oplus \operatorname{small}\left(G_{h}\right)$. Then $G$ is co-Hopfian (but not Hopfian if $x$ is epic).

Proof. To prove $G$ is co-Hopfian, let $f: G \rightarrow G$ be a monomorphism. We will show that it is an isomorphism. 
First, $f$ is of the form $f=\alpha+\phi$, where $\alpha \in J_{p}[[x]]$ and $\phi \in \operatorname{small}(G)$.

Let $n$ be a natural number. Since $\phi$ is small, there is a natural number $m$ such that

$$
p^{m} G\left[p^{n}\right] \subseteq \operatorname{ker} \phi,
$$

so $f$ coincides with $\alpha$ on $p^{m} G\left[p^{n}\right]$.

Consider the commutative diagram

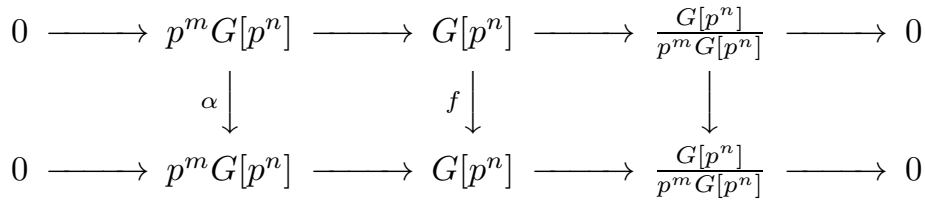

Since $f$ is monic, the left-most vertical arrow $\alpha$ is monic. Hence $\alpha$ must have a constant term not divisible by $p$, so $\alpha$ is invertible and therefore an isomorphism. It follows that the right-most vertical arrow is a monomorphism. As $G\left[p^{n}\right] / p^{m} G\left[p^{n}\right]$ is finite, this arrow must be an isomorphism. By the Five Lemma, therefore $f$ is an isomorphism on $G\left[p^{n}\right]$ for all $n$, and hence $f$ is also an isomorphism of $G$.

It is easy to see that $G_{c}$ is co-Hopfian by Lemma 3.1 and Proposition 3.2 As $x$ is an epimorphism but not a monomorphism, $G_{c}$ is not Hopfian, proving Theorem 1.1. (1.c)

\section{MARTIN's AXIOM AND Hopfian GROUPS}

In this section we show that $\mathrm{MA}(\sigma$-centered $)$ implies that every reduced infinite $p$-group $G$ of size less than $2^{\aleph_{0}}$ is neither Hopfian nor co-Hopfian, i.e., Theorem 1.1[ $(2)$

Let $G$ be a reduced $p$-group. We may assume that $G$ is semi-standard, as otherwise it is neither Hopfian nor co-Hopfian. Let $B$ be a basic subgroup of $G$.

We shall build endomorphisms of $G$ by gluing together partial endomorphisms defined on finite subgroups. The partial endomorphisms will be selected via Martin's Axiom. To ensure the countable chain condition, we voluntarily restrict to endomorphisms $f$ satisfying $G(f-1) \subseteq B$ so that every element of $G$ can be mapped to only countably many others. As a by-product, this also simplifies the presentation by allowing us to restrict to separable $G$, which we explain in the next two paragraphs.

The first Ulm factor $G / G^{(1)}$ is separable with a basic group isomorphic to $B$ via the natural map $B \rightarrow G / G^{(1)}$. By abuse of notation, let $B$ also denote this basic subgroup.

Now every endomorphism $f$ of $G / G^{(1)}$ with the image of $f-1_{G / G^{(1)}}$ in $B$ lifts uniquely to an endomorphism $\tilde{f}$ of $G$ which is the identity on $G^{(1)}$ and satisfies $G\left(\widetilde{f}-1_{G}\right) \subseteq B$. This is easily seen by considering $g=f-1_{G / G^{(1)}}$ and $\widetilde{g}=\widetilde{f}-1_{G}$ instead of $f$ and $\tilde{f}$ respectively, with the co-domain of $g$ and $\widetilde{g}$ restricted to $B$. Then the condition is that $\widetilde{g}$ is the composition of the natural projection $G \rightarrow G / G^{(1)}$ and $g$.

In particular, $\tilde{f}$ is a monomorphism or an epimorphism if and only if $f$ is so, respectively. This reduces the problem to separable groups $G$. 
Also note that because $f$ induces the identity on $G / B$, we derive analogously that $f$ is monic or epic, respectively, if so is the restriction $f: B \rightarrow B$. In particular, $f$ is epic if its image contains $B$.

From now on, let $G$ be an infinite semi-standard separable $p$-group of cardinality less than the continuum. In particular $G$ is unbounded. Let $B=\bigoplus_{n=1}^{\infty} B_{n}$ be a decomposition of the basic subgroup with $B_{n}$ being a direct sum of cyclic subgroups $\mathbb{Z}_{p^{n}}$ of order $p^{n}$. Let $H_{N}:=\left\langle p^{N} G, B_{N+1}, B_{N+2}, \ldots\right\rangle$ be the canonical direct complement of $\bigoplus_{n=1}^{N} B_{n}$ in $G$.

In the following subsections we construct a non-monic epic endomorphism and a non-epic monic endomorphism of $G$, respectively. Therefore $G$ is neither Hopfian nor co-Hopfian, as claimed.

4.1. Group is not Hopfian. To show that $G$ is not Hopfian, we apply Martin's Axiom to the following poset:

Elements: partial homomorphisms $g: H \rightarrow G$, where $H$ is a finite pure subgroup of $G$ with $H(g-1) \subseteq B$.

Order: $h \leq g$ if and only if $h$ is an extension of $g$.

Dense subsets:

- $\mathbb{D}_{x \in \text { dom }}:=\{g \mid x \in \operatorname{dom} g\}$ for $x \in G$,

- $\mathbb{D}_{b \in \text { im }}:=\{g \mid b \in \operatorname{im} g\}$ for $b \in B$,

- $\mathbb{D}_{\text {non-monic }}:=\{g \mid g$ not monic $\}$.

Filters: $\mathcal{F}_{\varphi}:=\{g \mid g \subseteq \varphi\}$ for $\varphi$ an endomorphism of $G$, which is the identity on some $H_{n}$ and $G(\varphi-1) \subseteq B$.

This is obviously a poset satisfying the conditions of $\mathrm{MA}(\sigma$-centered). E.g., there are only countably many filters $\mathcal{F}_{\varphi}$, as there are only countably many choices of $\varphi$ thanks to the restrictions $G(\varphi-1) \subseteq B$ and $\varphi\left\lceil H_{n}=1\right.$. These filters cover the poset, and as for any partial homomorphism $g: H \rightarrow G$, we can find a direct complement $C$ of $H$ in $G$ containing some $H_{n}$. Defining $\varphi$ to be $g$ on $H$ and the identity on $C$, we obtain $g \in \mathcal{F}_{\varphi}$. A similar but easier argument proves that the set $\mathbb{D}_{b \in \text { im }}$ is dense for $b \in B$. Now for $g: H \rightarrow G$, we choose a pure extension $K=H \oplus L$ with $L$ a cyclic direct summand of $B$ with sufficiently large order; thus $g$ extends to an $h: K \rightarrow G$ with $b \in L h \subseteq B$. It is even easier to show that the remaining sets claimed to be dense are really dense.

Applying $\mathrm{MA}(\sigma$-centered), there is a filter intersecting the dense subsets above. Taking the union of the homomorphisms in the filter, we obtain a non-monic endomorphism $f$ of $G$ with $G(f-1) \subseteq B$ and $B \subseteq G f$. It follows that $f$ is epic but not monic, indicating that $G$ is not Hopfian.

4.2. Group is not co-Hopfian. The construction is similar to the previous case with a suitable adjustment of the poset.

Let $0 \neq b_{0} \in B$ be a fixed non-zero element of $B$. Our aim is to construct a monic endomorphism of $G$ whose image does not contain $b_{0}$.

Elements: partial monomorphisms $g: H \rightarrow G$, where $H$ and the image $H g$ are finite pure subgroups of $G$ with $b_{0} \notin H g$ and $H(g-1) \subseteq B$.

Order: $h \leq g$ if and only if $h$ is an extension of $g$.

Dense subsets: $\mathbb{D}_{x \in \text { dom }}:=\{g \mid x \in \operatorname{dom} g\}$ for $x \in G$.

Filters: $\mathcal{F}_{\varphi}:=\{g \mid g \subseteq \varphi\}$ for $\varphi$ a monomorphism to $G$ defined on a subgroup of $G$ and extending the identity on some $H_{n}$. Moreover, the image of $\varphi-1$ lies in $B$ and $b_{0}$ is outside the image of $\varphi$. 
(For the filters, we allow $\varphi$ to be defined only on a subgroup, because a priori we cannot come up with a monic endomorphism of $G$ which is not epic. Actually, all the $\varphi$ we use will be restrictions of some isomorphism of $G$.)

Since $H_{n}$ is of finite index, there are only finitely many subgroups containing it, and hence there are altogether only countably many filters $\mathcal{F}_{\varphi}$.

To show that every partial monomorphism $g: H \rightarrow G$ is contained in an $\mathcal{F}_{\varphi}$, we proceed in several steps.

We choose decompositions $G=H \oplus C_{1} \oplus H_{n}$ and $G=H g \oplus C_{2} \oplus H_{n}$ for a suitable $n$ with $C_{1}, C_{2} \subseteq B$. By factoring with $H_{n}$, it follows that $H \oplus C_{1} \cong H g \oplus C_{2}$. As the groups involved here are finite, and $H \cong H g$ as $g$ is a monomorphism, by the fundamental theorem of abelian groups, the primary decomposition of $C_{1}$ and $C_{2}$ should coincide, hence $C_{1} \cong C_{2}$. We define $\varphi$ to be $g$ on $H$, an isomorphism to $C_{2}$ on $C_{1}$ and the identity on $H_{n}$. Hence $\varphi$ is an automorphism of $G$ extending $g$ and satisfying $G(\varphi-1) \subseteq B$. By restricting to a complement of an appropriate cyclic direct summand of $C_{1} \oplus H_{n}$, we can omit $b_{0}$ from the image of $\varphi$ with the domain still containing an $H_{m}$ for some $m \geq n$. Hence $g \in \mathcal{F}_{\varphi}$.

An easier argument shows that the $\mathbb{D}_{x \in \text { dom }}$ are dense, but care must be taken to omit $b_{0}$ from the image of the extension. For example, given $g: H \rightarrow G$, we can find decompositions $G=H \oplus C_{1} \oplus H_{n}$ with $x \in H \oplus C_{1}$, and $G=H g \oplus C_{2} \oplus H_{n}$ with $C_{2} \subseteq B$ for a suitable $n$. It follows that $H \oplus C_{1} \cong H g \oplus C_{2}$, and hence $C_{1} \cong C_{2}$.

We define the extension $h: H \oplus C_{1} \rightarrow G$ of $g$. Obviously, it should coincide with $g$ on $H$. On the other part, $C_{1}$, let $h$ be a modification of the inclusion of $C_{1}$ into $G=H g \oplus C_{2} \oplus H_{n}$ by replacing the $C_{2}$-component with an isomorphism $C_{1} \cong C_{2}$. Thus, $G=H g \oplus C_{1} h \oplus H_{n}$, hence $h$ is an extension of $g$ to an embedding onto a finite pure subgroup. The definition was carefully made to keep the image of $h-1$ inside $B$.

However, there is no guarantee that $b_{0}$ hasn't smuggled itself into the image. As a remedy, let's consider a variant $h^{\prime}$ of $h$ : since $H_{n} \cap B$ is reduced and unbounded, there is an embedding $\alpha: C_{1} \rightarrow H_{n} \cap B$. Now $h^{\prime}:=h+\alpha$ has the same properties as $h$ : it extends $g$, it embeds its domain into a finite pure subgroup, and the image of $h^{\prime}-1$ is in $B$. Moreover, $\operatorname{im} h \cap \operatorname{im} h^{\prime}=H g$, and therefore $b_{0}$ cannot be contained in the image of both $h$ and $h^{\prime}$, so at least one of them is an extension of $g$ in $\mathbb{D}_{x \in \text { dom }}$.

We conclude that the poset satisfies the conditions of MA $(\sigma$-centered). So there is a filter intersecting all the dense subsets above. The union of the monomorphisms of the filter produces a monic endomorphism $f$ of $G$ with $G(f-1) \subseteq B$ and $b_{0} \notin G f$. Hence $f$ is monic but not epic, showing that $G$ is not co-Hopfian.

\section{REFERENCES}

[B] Reinhold Baer, Groups without proper isomorphic quotient groups, Bull. Amer. Math. Soc. 50 (1944), 267-278. MR0009955 (5,228a)

[BP] R. A. Beaumont and R. S. Pierce, Partly transitive modules and modules with proper isomorphic submodules, Trans. Amer. Math. Soc. 91 (1959), 209-219. MR0107667 (21 \#6390)

[C] A. L. S. Corner, On endomorphism rings of primary abelian groups, Quart. J. Math. Oxford Ser. (2) 20 (1969), 277-296. MR0258949 (41 \#3594)

[Cr] Peter Crawley, An infinite primary abelian group without proper isomorphic subgroups, Bull. Amer. Math. Soc. 68 (1962), 463-467. MR0142646 (26 \#215)

[DGSZ] Dikran Dikranjan, Brendan Goldsmith, Luigi Salce, and Paolo Zanardo, Algebraic entropy for abelian groups, Trans. Amer. Math. Soc. 361 (2009), no. 7, 3401-3434, DOI 10.1090/S0002-9947-09-04843-0. MR2491886(2012b:37043) 
[F] L. Fuchs, Infinite Abelian Groups, Vol. I and II, Academic Press, 1970 and 1973. MR0349869

[G] Brendan Goldsmith, Anthony Leonard Southern Corner 1934-2006, Models, modules and abelian groups, Walter de Gruyter, Berlin, 2008, pp. 1-7, DOI 10.1515/9783110203035.1. MR 2513225

[GG] B. Goldsmith and K. Gong, On adjoint entropy of abelian groups, Comm. Algebra 40 (2012), no. 3, 972-987, DOI 10.1080/00927872.2010.543447. MR2899919

[GG2] B. Goldsmith and K. Gong, A note on Hopfian and co-Hopfian abelian groups, Groups and model theory, Contemp. Math., vol. 576, Amer. Math. Soc., Providence, RI, 2012, pp. 129-136, DOI 10.1090/conm/576/11356. MR2962880

[IW] John M. Irwin and Elbert A. Walker, On N-high subgroups of Abelian groups, Pacific J. Math. 11 (1961), 1363-1374. MR.0136653 (25 \#119a)

[P] R. S. Pierce, Homomorphisms of primary abelian groups, Topics in Abelian Groups (Proc. Sympos., New Mexico State Univ., 1962), Scott, Foresman and Co., Chicago, Ill., 1963, pp. 215-310. MR0177035 (31 \#1299)

Fakultät für Mathematik, Universität Duisburgessen, Campus Essen, 45117 Essen, GERMANY

Current address: H. Milton Stewart School of Industrial and Systems Engineering, Georgia Insitute of Technology, 755 Ferst Drive, NW Atlanta, Georgia 30332

E-mail address: gabor.braun@isye.gatech.edu

Faculty for Computer Sciences, Mannheim University of Applied Sciences, 68163 Mannheim, Germany

E-mail address: 1.struengmann@hs-mannheim.de 